\title{
The importance of youth perspectives on mental health
}

\author{
By Megan Franklin
}

In May 2014, Landon Pearson invited me to speak to issues contained in the document "A Canada Fit For Children," Canada's plan of action in response to the May 2002 United Nations Special Session, specifically focusing on Section C-Mental Health. There are three paragraphs $(94,95,96)$ from the Canada Fit for Children document that address mental health:

Paragraph 94 states, "A significant number of children in Canada have mental health issues that are serious enough to warrant clinical intervention. While some of these problems have physiological roots, family, school and community environments also have a profound influence. All children face challenges in their psychosocial development but most of them are surmountable problems that, in fact, help them to grow. However, if there are too many problems at once or if they are not resolved, then the child may find it difficult to adjust and becomes prone to dysfunctional relationships and to making unhealthy choices" (A Canada Fit for Children, 2004).

Paragraph 95 states, "All children benefit from opportunities to experience success, to make constructive choices, to understand emotions, and to share thoughts and feelings safely with one another and with adults. We in Canada will create and maintain the conditions that promote the mental health of children, young people and their families, and will strive to prevent or minimize the adverse consequences of emotional problems and mental illness. Depression in children is a growing concern. The suicide of young people, which is occurring too frequently in both Aboriginal and non-Aboriginal communities, is the ultimate tragedy; every effort must be made to prevent it" (A Canada Fit for Children, 2004). 
Paragraph 96 states. "We will make the promotion of mental and emotional health a key element of health promotion and protection strategies. We will recognize the interdependence of the generations and the critical role of community and family. We will raise awareness of the interactions between mental health and other pressing health and social issues, including gender, poverty and isolation. We will also promote understanding of health psychosocial development, respect the rights and dignity of persons experiencing emotional problems and mental illness, and reduce the stigma associated with mental health issues (A Canada Fit for Children, 2004).

As a current graduate student in the Social Work program at Carleton University, I am interested in young people's perspectives on issues concerning mental health because I believe that it is important to bring these perspectives to the attention of diverse audiences comprising doctors, child advocates, child protection lawyers, and politicians, among others. When young people are asked to speak to issues concerning mental health, they have much to say about their experiences in schools, families, and health care institutions. They have valuable insights into how identity and mental health issues come together in people's lives as well as what might be done to support and help young people more effectively.

I was honoured to have had the opportunity to share some of my experiences with the audience at the CRC anniversary event and to bring forward the fact that youth are still not being heard on matters concerning mental health as they should be. Having the opportunity to work with young people in various capacities as a graduate student researcher, and in talking with them about mental health and what the CRC says about the right to be heard, has been an important experience. It is also significant that most young people with whom I have spoken in the past few years have mentioned that they do not feel that they have been heard because of barriers including their age and social standing. The opportunity to reach a broad audience with these ideas further convinced me of the importance to get the message out that we need to 'tune in' with our youth and seek change through research and education. 